\title{
Bayesian Affordance-Based Agent Model for Wayfinding Behaviors in Evacuation Problems
}

\author{
Moise Busogi ${ }^{1}$, Namhun Kim ${ }^{1}$, Dongmin Shin $^{2}$, Hokyoung Blake Ryu ${ }^{2}$, \\ Arm Yoo ${ }^{1}$, and Dongchul Kim ${ }^{3}$ \\ ${ }^{1}$ Ulsan National Institute of Science and Technology, UNIST-gil 50, Ulsan 689-798, \\ Republic of Korea \\ \{busogi, nhkim, yoodkfmal\} @unist.ac.kr \\ ${ }^{2}$ Hanyang University, 222 Wangsimini-ro, Seongdong, Seoul 133-791, Republic of Korea \\ \{dmshin, hryu @ @hanyang.ac . kr \\ ${ }^{3}$ ITSTAR, 1327-6 Dal-dong, Nam-gu Ulsan, Republic of Korea \\ 4659issac@hanmail.net
}

\begin{abstract}
In this paper, we propose a modeling framework of rational human actions in human-environment systems by evaluating probable human actions in physical and psychological dimensions. In the affordance theoretic perspective, an environment offers certain physical and psychological limitations to filter a finite number of feasible human actions that lead to desired system states in a spatio-temporal dimension. By integrating physical and psychological constraints in human decision making processes, a value-based Bayesianaffordance model is proposed using Markov Decision Model. To this ends, two different types of filters, ' $F 1$ ' and ' $F 2$ ' are proposed, where ' $F 1$ ' is a preference-based numerical filter conceived at the planning level for psychological constraints and ' $F 2$ ' an affordance-based numerical filter at the execution level in which agent's perception of physical action availability plays a big role. Finally, a simple example based on the proposed model is illustrated to verify the proposed framework and the analysis results are discussed.
\end{abstract}

Keywords: Affordance, way-finding, dynamic programming, Markov Decision Process.

\section{Introduction}

Predicting human action is a challenging task, if not impossible due to many uncertain factors involving decision making of an agent [1]. However, the degree of difficulty can be tremendously reduced by acquiring more related information on the agent's perceptions and understanding of environments, such as its belief, its purpose as well as its environment [2].

According to Daniel Dennett, in his theory of intentional stance, predicting a rational agent is not somehow impossible task [2]. Predicting human action offers a boost in simulating human actions in space and time dimensions, which in turn provides a necessary tool to understand human-environment interactions. Gimblett et al. 1997 
explains how human action simulation not only allows examining but also testing different observation of a system behavior [3].

Despite many theories suggesting the unpredictability of human action, within a given space and time, the environment tend to offer plenty of constraints that give less and less options and choices to the agent, therefore making it easier to predict probable human actions to reach his/her goal. In some of human-environment interactions such as way-findings, there is a clear and a stable goal and human tend to perceive action opportunities and different cues in a somehow a consistent way. In this paper, action opportunities and constraints are modeled using the theory of affordance $[4,5]$ which will be discussed further in detail in section 2 .

Way-finding can be seen as a combination of human factors and environment factors where a human decision making is led by his or her perception of the environment. Arthur and Passini define wayfinding process as the combination of Decision making, Decision executing, and Information processing [6]. The wayfinding is heavily affected by the agent perceptual capabilities, fundamental informationprocessing capabilities, previously acquired knowledge, and motor capabilities [7].

This research intend to assist designers or modelers of human-involved systems in understanding and mapping human decision process with respect to the environmental affordances therefore helping them to create effective and efficient cues to guide human in making appropriate decision. Furthermore, due to limitations of human experimentation, the resulting agent will be very useful tool in simulating human behavior in an environment that was otherwise impossible or inappropriate for real human experimentation.

The rest of paper is composed as follows; in section 2,related works are briefly reviewed; in section 3, we present our model framework; in section 4, an example is given to illustrate the proposed framework; finally, a brief conclusion follows to wrap up the discussion.

\section{Related Works}

\subsection{Affordance Theory and Human Decision Modeling}

The word affordance was first introduced by Gibson in 1979 to refer to the environment property that provides or furnishes the animal to take an action, either for the good or ill of the system [4]. Since its introduction, the affordance theory got a lot of attention from researchers in various field such as artificial intelligent, usability, etc. [8-10], however, less has been done to incorporate it in human decision modeling. Joo et al. developed Conceptual Framework for Affordance-Based Simulation of Human Behavior using Finite State Automata (FSA) [11]. Whereas Joo et al. deeply consider the agent-environment interaction; there is no much focus on the agent abilities and preferences, therefore leading every agent in making exactly same choice. Chris et al. presented a Bayesian goal inference model, in which a Markov decision process was used to approximate action probabilities [12], however, among others, a systematic description of agent and environment interaction was given little attention. 


\section{$3 \quad$ Modeling Framework}

Rational agent are assumed to choose the most cost effective way to achieve a goal; however, limited information and different uncertainties within the agent environment makes it harder for the agent to effectively choose the optimal path to reach the intended goal. Due to these uncertainties, the agent makes a decision using the available information and updates his/her choices along the execution process when new information is revealed along the process [13].

To further understand, let's consider a system that consists of a human in a multistory building. In this system, we consider the floors as states of the system from which a human transit to another state (floor) by taking series of relevant actions. The actions can include: walking, running, taking an elevator, taking an escalator, jump, use a rope and so on. A rational agent choice of action usually correlates with the cost-effective way of reaching the goal. Agents encounter different types of costs while trying to reach a goal [14]. The computational model for representation of rational human agents should include the formal process of incurring a goal-directed and perception-based action as shown in Fig 1.

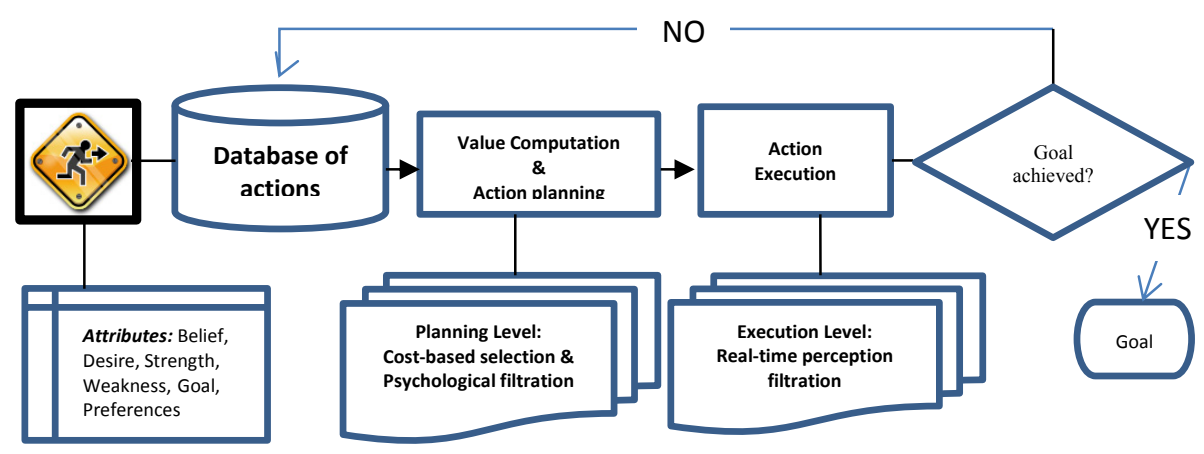

Fig. 1. Action selection process frameworkAction Selection- Physical Domain

We define an environment as anything not intrinsic to the agent, within the system. Within the system, there is an interaction between an agent and an environment. According to theory of affordance, this interaction is the essence of action and result in the changing of the agent's state. Agents are believed to have belief and desires [2]. These beliefs and desires differentiate one agent to another, and they do not only determine the agent's goal (g) but also its inner resources that lead the agent to choosing an action. As it is defined in the Markov decision process; our agent gets a reward $\mathrm{r}(\mathrm{t})$ for performing action $\mathrm{a}(\mathrm{t})$ and reaching state $\mathrm{s}(\mathrm{t}+1)$ from state $\mathrm{s}(\mathrm{t}) . \mathrm{C}(\mathrm{a})$ is the cost it takes an agent to take action a.

Using dynamic programming we assign optimal value to each state based on the optimal policy. A policy is a mapping of action to state. Since the agents are assumed to be rational, the probability of choosing an action is proportional to $\mathrm{Q}$, the expected value of taking the action. In the proposed model, the agents make a finite number of steps to reach the goal. Let $\pi$ be the best policy to reach the goal with the maximum 
total reward $r . \pi$ maps the series of actions that minimize total cost from the current state to the goal state, $\pi: s \in S \rightarrow \pi(s) \in A$, where $S$ denotes the set of states of the system, and A denotes the set of available actions. By following the optimum policy the value of state $\mathrm{s}$ will be

$V_{N}^{\pi}(s)=\sum_{t=0}^{t=N-1} E^{\pi}\left[r\left(s_{t}, a_{t} \mid s_{0}=s, g, w\right)\right]$

, where:

$V_{N}^{\pi}(s)$ : The expected value of state $s$ given that he executes policy $\pi$, $s_{t}:$ State at time $\mathrm{t}$,

$g$ : Agent's goal,

$w$ : Environment state,

$a_{t}$ : Agent action at time $\mathrm{t}$, and

$N$ : Finite number of steps to reach the goal.

While in state $s$, an agent can choose any available action within the state. To better understand the value of action a in state $\mathrm{s}, Q^{\pi}(s, a \mid g, w)$ is defined, which is the expected value when starting from $s$ executing action a and then follow policy $\pi$ afterwards. $Q^{\pi}(s, a \mid g, w)=r(s, a)+\sum_{s_{t}} p\left(s_{t} \mid s, a, g, w\right) V^{\pi}\left(s_{t}\right)$. Since we consider our agents to be rational at a given degree, the probability $P(a)$ to choose a given action in a given state will be proportional to its action-value of $Q^{\pi}(s, a \mid g, w)$ [15].

\subsection{Action Selection-Psychological Domain}

In 1956, Simon argued that people are satisfied with a good enough solution that approximate the accuracy of optimal algorithms without placing too heavy demand on the cognitive system [16], which mainly refers to the effect of psychological cost on human decision making. After rating different actions based on physical cost, the proposed model employs the psychological factors of preference as filters in the proposed framework of human action selection model. These filters use the subjective perception and interpretation of environmental affordances as well as the effectivity of the agent, not only in the planning level but also at the execution process.

Planning Level. The planning level filtration is an expected affordance-based psychological filtration of activities required to create a desired goal on some scale. By considering each action in the database of actions, each action is given a binary score $i$ with respect to both affordance and effectivities. For example, for an agent considering climbing stairs, his binary score on effectivity is 1 if he has physical ability to climb and the affordance score is 1 if there are stairs on which climbing is possible. If the stairs had missing steps, that makes it impossible for an agent to climb, the rating score would be 0 instead 1 for the affordance. It is important to understand that at the planning level, both affordance and effectivities are imaginary and approximated through different cues and prior experiences without a direct perception.

Among the possible actions, some are more affordable than others due to not only the energy or time spent performing these actions but also to different level of 
meditation [16]. In this sense, the meditation level is applied to model a function of stress, attention, or any other mental or emotional cost associated with taking any given action. Using the expected meditation level, the agent filters the available action using $F_{1}$, which is a numerical filter representing possibility to choose an action at the planning level. Let $p_{1}$ be a numerical score to denote the complexity of performing a given action a in a given environment. In other words, $p_{1}$ denote how mentally easy to perform a given action in a given environment within a given state. In the proposed model, $p_{1}$ takes a continuous value from 0 to 1 . Thus, more mentally challenging actions get relatively lower rating. The lower the stress, the higher the value of $p$, thus a stress-free state would mean $\mathrm{p}$ valued nearly to 1 . As specified earlier, $F_{1}$ is a product of not only the environment score but also the agent score with respect to a given action. In our model the agent score is denoted by $q_{1} . q_{1}$ denotes the agent relative level of expertise, skill, risk taking behavior and other subjective advantage or disadvantage in terms of taking a given action in a given state and a given environment state. Before determining the value of $q_{1}$ a reference agent should be determined by which other agents must be compared to. The reference agent should be a typical agent with characteristics of an average agent and its $q_{1}$ value should be 0.5 .

$$
F_{1}=i \cdot p_{1} \cdot q_{1} \text {, where }
$$

$$
\begin{aligned}
& i=\left\{\begin{array}{l}
1 \text { if the action is possible wrt to affordance Effectivities } \\
0 \\
\text { otherwise }
\end{array},\right. \text { and } \\
& \qquad q_{1}, p_{1} \in[0,1] .
\end{aligned}
$$

Incorporating the filter into the cost based selection gives us the new action probability.

$$
P(a)=P 1 . F 1 \text {. }
$$

Execution Level. An agent does not necessarily follow the path conceived in the planning level; instead, he/she may adapt to the real situation by updating his/her decision based on updated information. In the planning level, human agents expect specific action opportunities that might help them to reach the goal state in the imaginary spatial-temporal dimension. However, a real action opportunity of the affordance-effectivity dual is only available in a certain space and time. The expected environmental situation can change and it inevitably makes the agent change the plan to reach the goal state frequently. To account the change between the expected environment and the real environment, the agent applies the second filter ' $F_{2}$ ', which incorporates the agent's real-time perception of environmental affordance as opposed to the expected affordance in the planning level. When the expected action opportunity conceived at the planning level matches the real time affordance, the agent should stick to the decision made in the planning level. Therefore $F_{2}$ must penalize any action for which the existence of affordance-effectivity dual is less probable than expected. Also, $F_{2}$ must promote the actions for which the affordance-effectivity duals are more probable than expected. Similarly, $F_{2}$ is defined as follow:

$$
F 2=\text { i. } \mathrm{p}_{2} \cdot \frac{\mathrm{p}_{2}}{\mathrm{p}_{1}}, \text { where }
$$




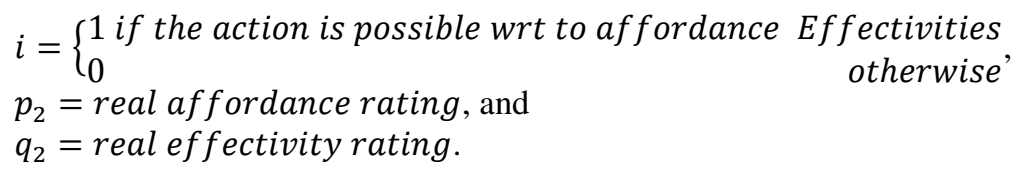

Let $P(a)$ denote the probability of a given action at the planning level. $P^{\prime}(a) \sim P(a) . F_{2}$, where $P^{\prime}(a)$ is the action probability at the execution level.

\section{$4 \quad$ An Illustrative Example}

For verification of the proposed model, we illustrate two different environment states separately in the analysis of the multi-story building.

\subsection{Optimum Policy}

In this illustration we consider a policy as any combination of action-state that result in reaching the goal. For example, for an agent in 4th floor whose goal is to exit the building, taking an elevator to the first floor and then exit the building can be considered as one of the policies. Fig 2 illustrates few possible policies. A policy is optimal if and only if it reaches the goal while maximizing total rewards; in our case, minimizing the total cost of reaching the goal.

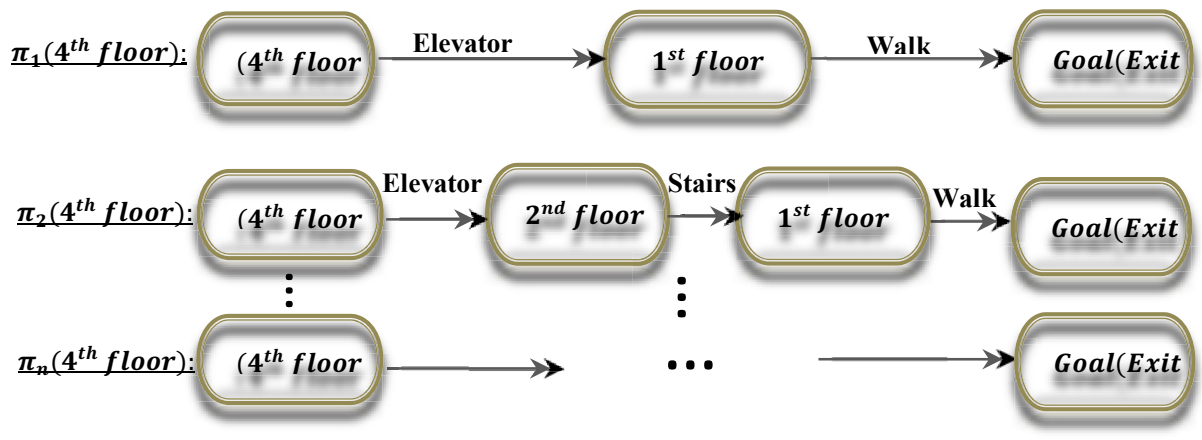

Fig. 2. Policy options

For each policy $\pi_{i}(\mathrm{~s})$ there is an associated value $V^{\pi}(s)$ which is the function of rewards collected throughout the process to reach the goal. In this illustration we express the rewards in function of the total cost of reaching the goal following cy $\pi_{i}(\mathrm{~s})$. $\quad V^{\pi}(s)$ is inversely proportional to the cost function. Let $c_{i}$ be the cost of reaching the goal following the policy $\pi_{i}(\mathrm{~s}) \cdot \pi_{i}(\mathrm{~s})=\pi_{\text {optimal }}(\mathrm{s})$ if and only if $c_{o p t}=\operatorname{Min}\left(c_{i}\right)$ for all $i$. In this stage, dynamic programing algorithm is used to assign the optimal policy to each state as well as the associated values. Based on the optimal values of each state obtained following a given policy, the agent compute 
$Q^{\pi}(s, a \mid g, w)$ which is the expected value when starting from state, $s$, executing action a and then follow policy $\pi_{i}\left(s^{\prime}\right)$ afterwards.

\subsection{Normal Working Environment}

We consider the building floors as state of the system and the exit being the final state which is the goal in this case. First of all, we compute the cost of taking a given action from a given state in a given environment. Only three states are considered in this example: 5th floor, 1st floor and goal which is to exit the building. The following table shows the energy cost as well as the time required to perform a given action from 5 th floor. It was computed based on little references ${ }^{1}$ and intuition.

Table 1. Cost of action

\begin{tabular}{|l|c|c|c|c|c|}
\hline \multicolumn{1}{|c|}{ Action } & Time & Energy & Cost & $\boldsymbol{p}\left(\mathbf{s}^{\prime} /\right.$ & $\boldsymbol{P 1}(\boldsymbol{a} / \boldsymbol{g}, \boldsymbol{s}, \boldsymbol{w})$ \\
\hline Take stairs & $1 \mathrm{~min}$ & $10 \mathrm{Cal}$ & 2 & 1 & 0.089766607 \\
\hline Take elevator & $30 \mathrm{sec}$ & $1 \mathrm{Cal}$ & 0.1 & 1 & 0.897666068 \\
\hline jump & $5 \mathrm{sec}$ & $1 \mathrm{Cal}$ & 0.016667 & 0.001 & 0.010771993 \\
\hline Use a rope & $2 \mathrm{~min}$ & $200 \mathrm{Cal}$ & 80 & 0.8 & 0.001795332 \\
\hline
\end{tabular}

Since time and energy have different units, it is hard to add up the total cost in order to compare different actions. A clear research on agent indifference curve for time and energy is needed to better decide their weight in the cost function. In this illustration, the cost is the product of both time and energy where 30 seconds of time and 10 calories of energy are both equivalent to one unit of cost. Using the cost value we compute the $Q^{\pi}$ value of every available action. The reward of performing an action is proportional to the probability of reaching the next state and inversely proportionally to the cost. The action probabilities based on the physical cost of performing the action are stored in Table 1. As discussed earlier, psychological cost also play a major role in this probability distribution, as shown in Table 2.

Table 2. Psychological filters -Normal environment

\begin{tabular}{|l|c|c|c|c|}
\hline Action & $\boldsymbol{p 1}$ & $\boldsymbol{i}$ & $\boldsymbol{q} \mathbf{1}$ & $\boldsymbol{F l}$ \\
\hline Take elevator & 0.9 & 1 & 0.5 & 0.4 \\
\hline Walk Stairs & 0.8 & 1 & 0.5 & 0.45 \\
\hline Jump & 0.01 & 1 & 0.5 & 0.005 \\
\hline Use a rope & 0.3 & 1 & 0.5 & 0.15 \\
\hline
\end{tabular}

Recall that $\mathrm{p} 1$ denotes how mentally easy to perform a given action in a given environment whereas q1 denote the agent relative level of expertise, skill, risk taking behavior and other subjective advantage or disadvantage in terms of taking a given action in a given state and a given environment state. Note that the ratings given in

${ }^{1}$ http: //www.healthstatus. com/calculate/cbc 
Table 2 are for a reference agent. The ratings are intuitive in this illustrative example. By using our model framework, the action probabilities are depicted in the chart below. Despite being hard to know the real probability in real life situation, it is still understandable to assume that more than 90 percent of people in 5 th floor will plan to take an elevator when they are trying to exit the building. At the execution level, the probability may change depending on the real situation. For example if there are many people waiting for the elevator, some people may opt to take stairs instead of waiting for the elevator. Similarly, in case of unexpected emergency like fire, F2 must be used to adjust the new and real situation as discussed in the next section.

\subsection{Evacuation in Emergency}

In case of fire emergency, an agent must figure out the right action given the urgent and dangerous environment. While taking an elevator was safe in the normal situation, in case of emergency, taking an elevator, becomes more risky, which explains why $F 1$ in both environment must differ as shown in Table 3, since the underlying psychological cost is different.

Table 3. Psychological filters -Fire emergency

\begin{tabular}{|l|l|l|l|l|}
\hline Action & $\boldsymbol{p 1}$ & $\boldsymbol{i}$ & $\boldsymbol{q 1}$ & $\boldsymbol{F 1}$ \\
\hline walk the stairs & 0.8 & 1 & 0.5 & 0.4 \\
\hline take an elevator & 0.2 & 1 & 0.5 & 0.1 \\
\hline jump & 0.1 & 1 & 0.5 & 0.05 \\
\hline Use a rope & 0.4 & 1 & 0.5 & 0.2 \\
\hline
\end{tabular}

As shown in Fig 3, in case of fire emergency, the majority of people obviously use the stairs as expected. In emergency situation $F 2$ plays a major role since there is a big gap between information available to the agent at the planning level and the realtime information. For example, the location of the fire and its propagation speed will certainly affect the agent action selection and $F 2$ ensures that the real-time information is incorporated into the proposed model. $F 2$

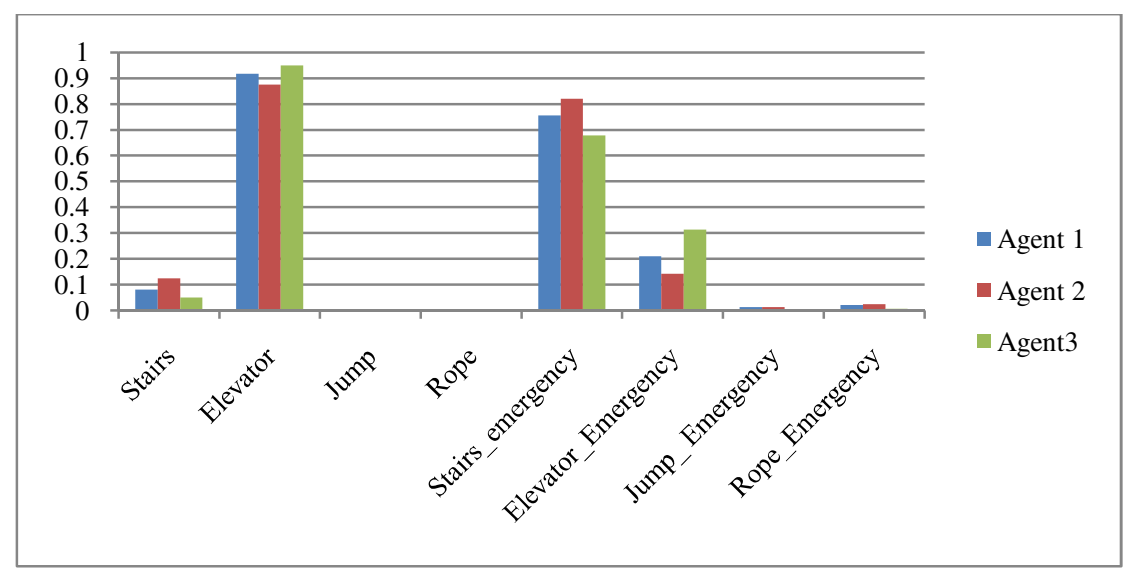

Fig. 3. Action probability 


\section{Conclusion}

Although affordance theory has explained much of goal-directed and perceptionbased human actions within environments, it has been a different story to build a computational model based on the theory of affordance to represent human behavior in the system. Thus, in this paper, we propose a Bayesian affordance-based model for human decision making behavior for representation of a rational agent. The model uses MDP to estimate action-state values for the optimal policy while different affordance-based filters are used to capture the possible actions at the planning level as well as the execution level. In the execution level of the action taking/decision making process of the human agent model, the state transitions (human actions) are probabilistically occurred based on the state values as well as series of affordance-based numerical filters which are a preference-based numerical filter of $F 1$ in planning level and physical filter of $F 2$ in the execution level. The model is expected to mimic rational human behaviors and to be used for agent-based modeling and simulation of human-included complex systems. From the planning level to the execution level, the proposed framework covers dynamic decision making processes with consideration of MDP for planning level and affordance-based model for execution level. We adopt the probability based action selection model to present the uncertainty of human actions within the dynamic environments.

A simple numerical illustration has been used to explain our model. The illustrative example shows how the proposed framework can work with MDP and affordance concept in the planning and execution levels. We confess that the model has not been validated and tested in real situation. However, we still think that the proposed model can be used to investigate the influence of human-involvement into the system and show the effect of interactions among the system and human agents under dynamic and uncertain situations. In this regard, we are planning to build a more sophisticated illustrative example using agent-based simulation, and validate it to the real life situation (e.g., building evacuation under fire) in the future.

Acknowledgement. This research was supported by Basic Science Research Program through the National Research Foundation of Korea (NRF) funded by the Ministry of Education, Science and Technology (No 2012-0003593 and No. 2012-047663).

\section{References}

1. Whitely, S.E.: Individual inconsistency: Implications for test reliability and behavioral predictability. Applied Psychological Measurement 2(4), 571-579 (1978)

2. Dennett, D.C.: The Intentional Stance (6th printing). MIT Press, Cambridge (1996)

3. Gimblett, H., Itami, R., Durnota, D.: Some Practical Issues in Designing and Calibrating Artificial Human-Recreator Agents in GIS-based SimulatedWorlds. Complexity International 3 (1996)

4. Gibson, J.J.: The Ecological Approach to Visual Perception. Houghton Mifflin, Boston (1979) 
5. McGrenere, J., Ho, W.: Affordances: Clarifying and Evolving a Concept. In: Proceedings of Graphics Interface Montreal, Quebec, Canada, pp. 179-186 (2000)

6. Passini, R.: Wayfinding: A conceptual framework. Urban Ecology 5(1), 17-31 (1981)

7. Allen, G.L.: Spatial abilities, cognitive maps, and wayfinding: Bases for individual differences in spatial cognition and behavior, pp. 46-80. Johns Hopkins University Press, Baltimore (1999)

8. Arkin, R.C.: Behavior-based robotics. MIT Press, Cambridge (1998)

9. Kirlik, A., Miller, R.A., Jagacinski, R.J.: Supervisory control in a dynamic uncertain environment: A process model of skilled human-environment interaction. IEEE Transactions on Systems, Man, and Cybernetics 23(4), 929-952 (1993)

10. Kim, N., Shin, D., Wysk, R., Rothrock, L.: Using finite state automata (FSA) for formal modeling of affordances in human-machine cooperative manufacturing systems. International Journal of Production Research 48(5), 1303-1320 (2010)

11. Joo, J., Kim, N., Wysk, R., Rothrock, L., Son, Y., Oh, Y., Lee, S.: Agent-based simulation of affordance-based human behaviors in emergency evacuation. Simulation Modeling Practice and Theory 32, 99-115 (2013)

12. Baker, C.L., Tenenbaum, J.B., Saxe, R.R.: Goal inference as inverse planning. In: Proceedings of the Twenty Ninth Annual Conference of the Cognitive Science Societ (2007)

13. Ariely, D.: Controlling the Information Flow: Effects on Consumers' Decision Making and Preferences. Journal of Consumer Research 27(2), 233-248 (2000)

14. Dubberly, H., Maupin, C.J., Pangaro, P.: Bio-cost: An Economics of Human Behavior. Guest Column in ASC / Cybernetics of Human Knowing (2010)

15. Sigaud, O., Buffet, O.: Markov Decision Processes in Artificial intelligent. John Wiley \&Sons, Inc., Hoboken (2010)

16. Simon, H.A.: Rational choice and the structure of environments. Psychological Review 63, 129-138 (1956) 